\title{
Model Predictive Control of Thermal Energy Storage in Building Cooling Systems
}

\author{
Yudong $\mathrm{Ma}^{\star}$, Francesco Borrelli`, Brandon Hencey ${ }^{\diamond}$, Andrew Packard ${ }^{\star}$, Scott Bortoff ${ }^{\diamond}$
}

\begin{abstract}
A preliminary study on the application of a modelbased predictive control (MPC) of thermal energy storage in building cooling systems is presented. We focus on buildings equipped with a water tank used for actively storing cold water produced by a series of chillers. Typically the chillers are operated each night to recharge the storage tank in order to meet the buildings demand on the following day. A MPC for the chillers operation is designed in order to optimally store the thermal energy in the tank by using predictive knowledge of building loads and weather conditions. This paper addresses real-time implementation and feasibility issues of the MPC scheme by using a (1) simplified hybrid model of the system, (2) periodic robust invariant sets as terminal constraints and (3) a moving window blocking strategy.
\end{abstract}

\section{INTRODUCTION}

According to the statistics complied by Environmental Protection Agency (EPA) in 2004, buildings in the United States account for $39 \%$ of total energy usage, $12 \%$ of the total water consumption, $68 \%$ of total electricity consumption, and $38 \%$ of the carbon dioxide emissions. It is therefore economically, socially and environmentally significant to reduce the energy consumption of buildings.

Reductions of $70 \%$ in energy use in buildings are required to achieve the goals for the building sector set by a number of organizations, including the California Public Utilities Commission. Achieving this goal requires the development of highly efficient heating and cooling systems, which are more challenging to control than conventional systems [1], [2], [15], [14], [6]. For a wide range of innovative heating and cooling systems, their enhanced efficiency depends on the active storage of thermal energy.

This paper focuses on the modeling and the control of the thermal energy storage on the campus of the University of California, Merced, USA. The campus has been designed to be a "living laboratory" and has a significantly enhanced level of instrumentation in order to support the development and demonstration of energy-efficient technologies and practices. It consists of a chiller plant (three chillers redundantly configured as two in series, one backup in parallel), an array of cooling towers, a $7000 \mathrm{~m}^{3}$ chilled water tank, a primary distribution system and secondary distribution loops serving each building of the campus. The two series chillers are operated each night to recharge the storage tank which meets campus cooling demand the following day. Although the

\footnotetext{
* Y. Ma, A. Packard, F. Borrelli are with the Department of Mechanical Engineering, University of California, Berkeley, CA 94720-1740, USA. Email: myd07, fborrelli, apackard@berkeley.edu.

B. Hencey and S. Bortoff are with United Technologies Research Center, East Hartford, Connecticut, USA. Email: HenceyBM, BortofsA@utrc.utc.com.
}

storage tank enables load shifting to off-peak hours to reduce peak demand, the lack of an optimized operation results in conservatively over-charging the tank where conductive losses erode efficiency. The objective of this paper is to design a predictive controller in order to minimize energy consumption while satisfying the unknown but bounded cooling demand of the campus buildings and operational constraints.

The main idea of predictive control is to use the model of the plant to predict the future evolution of the system [7], [8], [13]. At each sampling time, starting at the current state, an open-loop optimal control problem is solved over a finite horizon. The optimal command signal is applied to the process only during the following sampling interval. At the next time step a new optimal control problem based on new measurements of the state is solved over a shifted horizon. The resultant controller is referred to as Receding Horizon Controller (RHC). A receding horizon controller where the finite time optimal control law is computed by solving an on-line optimization problem is usually referred to as Model Predictive Control (MPC). For complex constrained multivariable control problems, model predictive control has become the accepted standard in the process industries [5]: its success is largely due to its almost unique ability to handle, simply and effectively, hard constraints on control and states.

Although this paper focuses on the specific architecture of the UC Merced Campus, the main ideas and methodologies can be applied to a wider class of buildings systems which use thermal energy storage. In particular our contributions are: (i) the development of a simple, yet descriptive bimodal switching nonlinear model of the overall cooling system, (ii) the development of a MPC scheme for minimizing energy consumption, (iii) the presentation of preliminary simulation results showing a potential $10 \%$ energy saving compared to currently adopted policies.

Particular attention is given to the real-time implementation and feasibility issues of MPC:

- A dual stage optimization is used in order to efficiently solve a mixed-integer nonlinear program [16], [3]: the first stage selects when to charge the tank by incorporating heuristics on the plant operation and its cost; the second stage optimally controls the chillers during the charging period. In the second stage a periodic moving window blocking strategy is used in order to reduce the computational time associated with the resulting nonlinear constrained optimization.

- Persistent feasibility requires that the tank has always enough energy to satisfy an unknown but bounded time- 
vary building cooling demand. Persistent feasibility is obtained in our scheme by using a time-varying periodic robust invariant set as terminal constraint. Such periodic invariant sets are computed base on the algorithm proposed in [4] and on experimental data.

We remark that the evaluation of optimal controllers for active and passive building thermal storage has been studied in the past by several authors (see [9], [17], [11], [10] and references therein). In particular in [9] the authors investigate a three-story office building equipped with two chillers with constant coefficient of performance and a thermal energy storage system. An optimal controller is designed in order to compute the cooling produced by the two chillers without accounting for pump power. Experimental results are presented in [17] where the optimal controller is implemented in a receding horizon fashion on an unoccupied test bed.

The paper is organized as follows. In Section II a general introduction to the system, and develops a simplified hybrid model of the system. In Section III the MPC control algorithm is outlined together with the dual stage strategy, the move blocking strategy as well as the terminal constraints computation. Simulation results are presented in Section IV. Finally, conclusions are drawn in Section V.

\section{SYSTEM MODEL}

In this section we describe the main components of the UC Merced Campus used to generate and store thermal energy. UC Merced campus has been built with a vision to create living laboratories for energy research. In this paper we focus on the higher level control systems which actuates three electric chillers that are operated at night to take advantage of nighttime electricity rates and lower ambient temperature when filling up two-million-gallon tank of chilled water. The following day, the chilled water is pumped from the tank and distributed throughout the campus. Secondary pumps draw water from the distribution system into each building, where it runs through a set of air-handler units (AHUs) and returns to the tank. Figure 1 shows the main scheme of the system.

The system consists of a condenser loop, a primary loop, a secondary (campus) loop, and several tertiary (building) loops. The chilled water is generated via chillers and cooling towers within the the primary and condenser loops. The chilled water is stored in a stratified thermal energy storage tank. The chilled water is distributed to the buildings throughout campus via the secondary loop. The tertiary loop uses pumps and valves within each building to distribute the chilled water for consumption by the cooling coils and air handling units (AHUs). The chilled water is warmed by the air-side cooling load of the buildings and returned to the secondary loop.

The next section presents a dynamic model of the system. Our objective is to develop a simplified yet descriptive model which can be used for real time optimization in a MPC scheme.

\begin{tabular}{cl}
\hline parameters & description \\
\hline$\rho:$ & fluid density $\left[\mathrm{kg} / \mathrm{m}^{3}\right]$ \\
$A_{c}:$ & cross sectional area of tank $\left[\mathrm{m}^{2}\right]$ \\
$k_{1}:$ & heat transfer coefficient to ambient $[\mathrm{W} / \mathrm{K}]$ \\
$k_{2}:$ & heat transfer coefficient between \\
& cool and warm mass of water $[\mathrm{W} / \mathrm{K}]$ \\
$C_{p}:$ & specific heat of water $\left[\mathrm{J} /{ }^{\circ} \mathrm{C}\right]$ \\
$r_{\operatorname{tank}}:$ & radius of the tank $[\mathrm{m}]$ \\
$S:$ & Weight on the energy consumption \\
$R:$ & Weight on the control input \\
$H_{p}:$ & Length of prediction horizon \\
$H_{u}:$ & Length of control horizon \\
\hline
\end{tabular}

TABLE I: Parameters

\begin{tabular}{cl}
\hline variables & description \\
\hline$h:$ & specific enthalpy $[\mathrm{J} / \mathrm{kg}]$ \\
$\dot{H}:$ & enthalpy flow rate $[\mathrm{W}]$ \\
$\dot{m}:$ & mass flow rate $[\mathrm{kg} / \mathrm{s}]$ \\
$p:$ & pressure $[\mathrm{Pa}]$ \\
$P:$ & power $[\mathrm{W}]$ \\
$U:$ & Internal energy of water $[\mathrm{J}]$ \\
$\dot{Q}:$ & heat flow transferred from one \\
& medium to another $[\mathrm{W}]$ \\
$T:$ & Temperature $[\mathrm{K}]$ \\
$m:$ & mass of water \\
$\dot{Q_{l}}:$ & height of water $[\mathrm{m}]$ \\
& cooling load $[\mathrm{W}]$ \\
&
\end{tabular}

\begin{tabular}{cl}
\hline subscriptions & description \\
\hline$\cdot b:$ & water below thermocline \\
$\cdot a:$ & water above thermocline \\
$\cdot{ }^{c} m p:$ & campus \\
${ }^{c} m p, r:$ & campus return \\
$\cdot{ }^{c} m p, s:$ & campus supply \\
$\cdot C H W S:$ & chilled water supplied from the chillers \\
$\cdot C H W R:$ & chilled water returning to the chillers \\
$\cdot a m b:$ & ambient \\
$\cdot{ }_{w b}:$ & wet bulb \\
\hline
\end{tabular}

TABLE III: Subscripts

\section{A. Nomenclature}

The following variables, parameters and subscripts will be used in this paper.

\section{B. Simplifying Assumptions}

[A1] The water in the tank is subject to minor mixing and thus can be modeled as a stratified system with layers of warmer water $(285 \mathrm{~K})$ at the top and cooler water $(277 \mathrm{~K})$ at the bottom. Figure 2 depicts the temperature of the water measured inside the tank at different heights at 8:30am on the 29th of Nov. 2007. One can observe a steep temperature gradient over the height of the tank, which is known as a thermocline. For this reason we lump warmer water above the thermocline and cooler water below the thermocline to obtain a 4-state system describing the height and temperature of the warmer and cooler water, respectively. Note that in this paper cooler (warmer) water means water that is cooler (warmer) than the thermocline. 


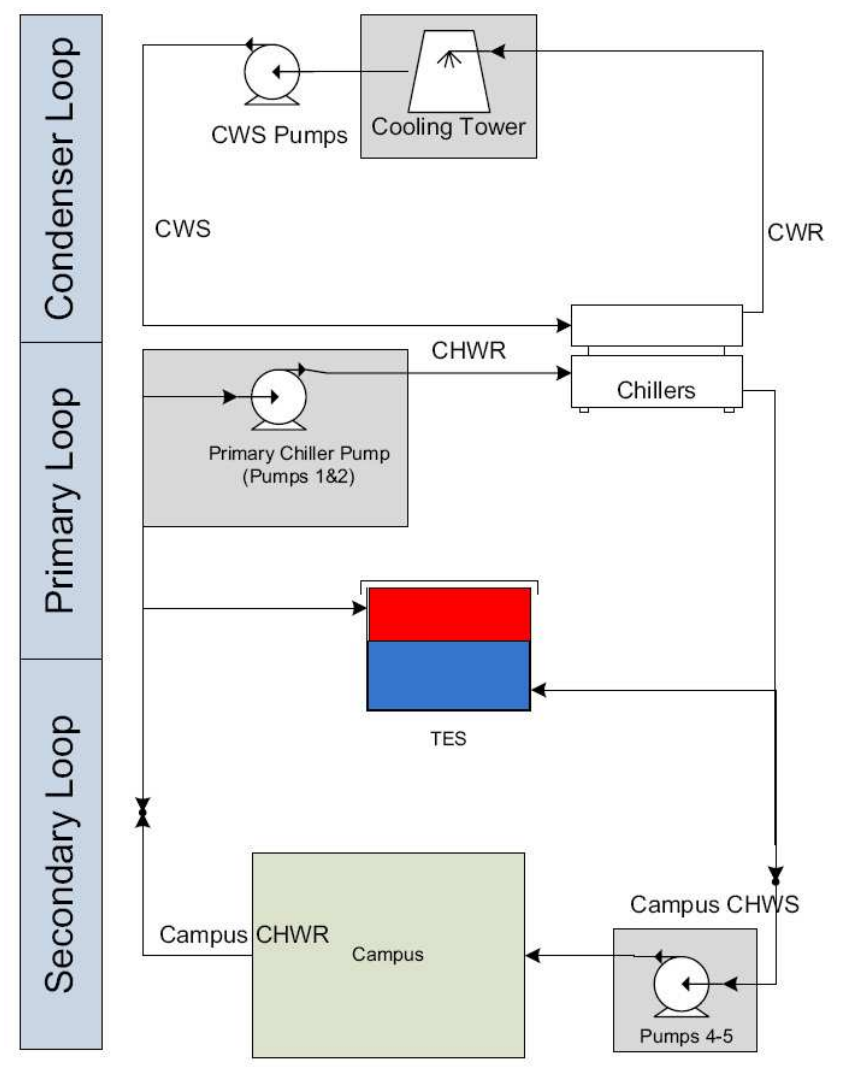

Fig. 1: Scheme plot of the chilling system

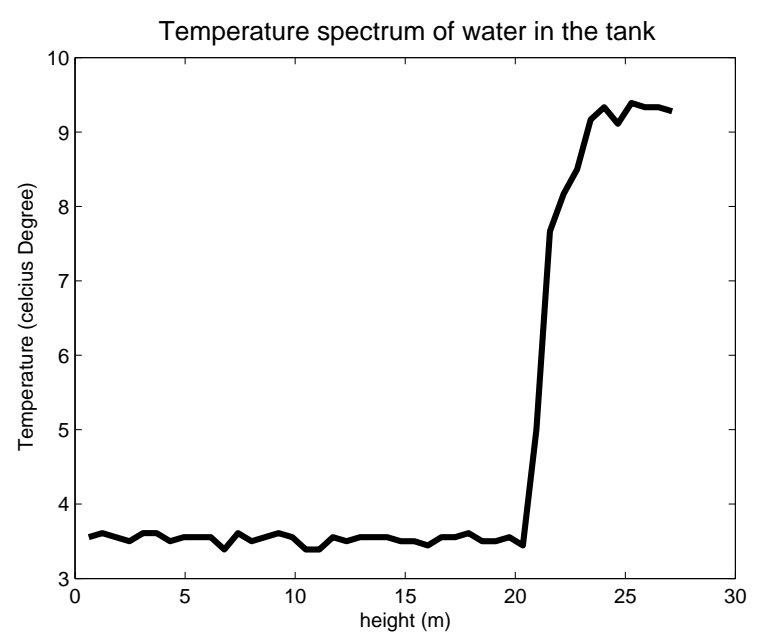

Fig. 2: Temperature distribution of water in the tank

[A2] Lower-level controllers actuate chillers and cooling towers in order to achieve a desired temperature of condensed water produced by cooling towers $T_{C W S \text {, ref }}$, mass flow rate of chilled water supplied by chillers $\dot{m}_{C H W S, r e f}$ and chilled water temperature $T_{C H W S, r e f}$. We neglect the dynamics of controlled chillers and cooling towers and assume that there is no tracking error between controlled variables and references so that:

$$
\begin{aligned}
& T_{C W S}=T_{C W S, r e f} \\
& \dot{m}_{C H W S}=\dot{m}_{C H W S, r e f} \\
& T_{C H W S}=T_{C H W S, r e f}
\end{aligned}
$$

[A3] Pipes are frictionless and insulated.

[A4] The campus is considered as a lumped disturbance in terms of heat flux required to cool down all buildings over the campus.

\section{Main subsystems of the cooling system}

Next we detail the main system components and their models.

1) Chillers and Cooling Towers Model: Based on assumption A2, the power used by the pumps, chillers and the cooling towers is modeled as a static function of $T_{C H W S}$, $T_{C W S}, \dot{m}_{C H W S}, T_{C H W R}$ (defined in assumption A2):

$$
P=\operatorname{Power}\left(T_{C H W S}, T_{C W S}, \dot{m}_{C H W S}, T_{w b}, T_{C H W R}\right) .
$$

where $T_{w b}$ is the temperature read from a wet bulb thermometer. The wet bulb temperature physically reflects the temperature and humidity of the ambient air.

The function Power(.) is implemented as a 5-D look-up table $(7 \times 6 \times 6 \times 5 \times 7)$ obtained by extensive simulations of a high fidelity model under various initial conditions.

2) Thermal Energy Storage Tank: We use assumption A1, and also assume that the tank is part of a closed hydronic loop, that is, the mass flow rate entering (exiting) the tank is equal to the mass flow rate exiting (entering) the tank. Subsequently, the height of water in the tank $z_{a}+z_{b}$ is a constant $z_{\text {tank }}$.

The tank can operate in two modes depending on the control inputs and the disturbances.

a) Charging: If the flow rate produced by the chiller is greater than campus flow rate $\dot{m}_{c m p, s}$, the difference will be charging the tank. By simple mass and energy conservation law, the dynamic of the tank in charging mode can be modeled as:

$$
\begin{aligned}
& \dot{H}_{b}=\dot{m}_{b} C_{p} T_{C H W S} \\
& \dot{H}_{a}=\dot{m}_{a} C_{p} T_{a} \\
& T_{c m p, s}=T_{C H W S} \\
& T_{C H W R}=\frac{T_{c m p, r} \dot{m}_{c m p, r}-T_{a} \dot{m}_{a}}{\dot{m}_{C H W R}} \\
& \dot{m}_{C H W S} \geq \dot{m}_{c m p, s}
\end{aligned}
$$

where the variables have been defined in Tables I, II, III.

b) Discharging: If the flow rate produced by the chiller is less than campus flow rate $\dot{m}_{c m p, s}$, tank will be discharged. The following equations model the dynamic of the tank in 


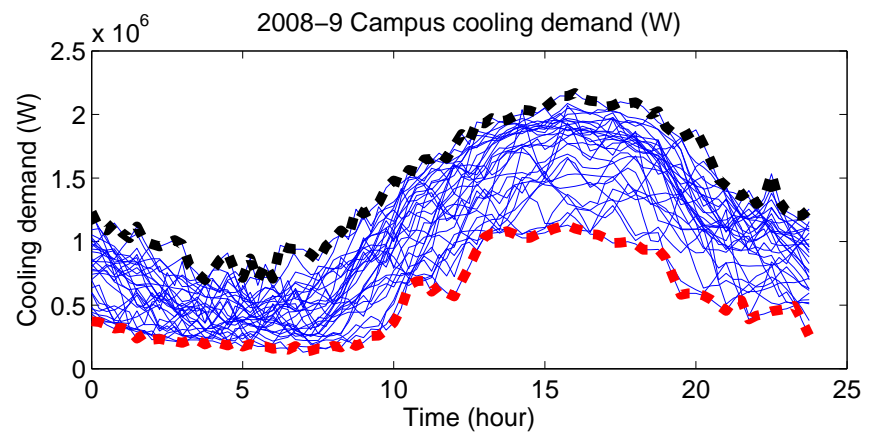

Fig. 3: Campus Load

charging mode.

$$
\begin{aligned}
& \dot{H}_{a}=\dot{m}_{a} C_{p} T_{c m p, r} \\
& \dot{H}_{b}=\dot{m}_{b} C_{p} T_{b} \\
& T_{c m p, s}=\frac{T_{C H W S} \dot{m}_{C H W S}-T_{b} \dot{m}_{b}}{\dot{m}_{c m p}} \\
& T_{C H W R}=T_{c m p, r} \\
& \dot{m}_{C H W S} \leq \dot{m}_{c m p, s}
\end{aligned}
$$

Independently of the mode, the mass and internal energy conservation laws always hold:

$$
\begin{aligned}
& \dot{z}_{b}=\left(\dot{m}_{C H W S}-\dot{m}_{c m p, s}\right) / \rho / A_{c} ; \\
& \dot{z}_{a}+\dot{z}_{b}=0 ; \\
& \dot{U}_{a}=\dot{H}_{a}+\dot{Q}_{b>a}+\dot{Q}_{A m b>a} ; \\
& \dot{U}_{b}=\dot{H}_{b}+\dot{Q}_{a>b}+\dot{Q}_{A m b>b} ;
\end{aligned}
$$

Where $\dot{Q}_{A m b>a}\left(\dot{Q}_{A m b>b}\right)$ is the heat transfer from ambient to the warmer (cooler) water in the tank:

$$
\dot{Q}_{A m b>a}=\left(T_{a m b}-T_{a}\right)\left(2 \pi r_{t a n k} z_{a}\right) k_{1}
$$

and $\dot{Q}_{a>b}\left(\dot{Q}_{b>a}\right)$ is the heat conducted from warmer (cooler) water to cooler (warmer) water in the tank:

$$
\dot{Q}_{a>b}=\left(T_{a}-T_{b}\right)\left(\pi r_{\text {tank }}^{2}\right) k_{2}
$$

3) Campus Model: We use assumption A4 along with a simple energy balance equation, and the campus load can be represented as:

$$
\dot{Q}_{c m p}=\dot{m}_{c m p} C_{p}\left(T_{c m p, r}-T_{c m p, s}\right)
$$

Where $\dot{m}_{c m p}$ is mass flow rate to the campus; $T_{c m p, s}$ is the temperature of water supplied to the campus; $T_{c m p, r}$ denotes the temperature of water returning to the campus; $\dot{Q}_{c m p}$ is the summation of the heat load required from each campus building. We use historical data of $T_{c m p, s}, T_{c m p, r}$ and $\dot{m}_{c m p}$ in order to compute the possible range of $\dot{Q}_{c m p}$. Figure 3 plots historical daily campus load during Sep. 2008, and we observe that the load has a period of one day. It is reasonable to model the load as a periodic disturbance with periodic envelope constraints (the bounds are represented with thicker lines in Figure 3).

\section{Control Variables}

1) $T_{C W S, r e f}$ : Reference temperature of the water exiting the cooling tower.

2) $\dot{m}_{C H W S}$ : Mass flow rate of the chiller water supply. It is a disconnected set. The mass flow rate is 0 when chiller is off, and $[2,235]$ while the chiller is operating.

3) $T_{C H W S, r e f}$ : Reference temperature of the water out of the chiller.

\section{E. Measured Variables}

1) $T_{C H W R}$ : Temperature of the water flowing back to the chiller.

2) $T_{a}$ : Temperature of the cool water in the tank.

3) $T_{b}$ : Temperature of the warm water in the tank.

4) $Z_{a}$ : Height of the warm water in the tank above the thermocline.

5) $Z_{b}$ : Height of the cool water in the tank below the thermocline.

\section{F. Operation Constraints}

The following constraints avoid the malfunction of the system components.

- $T_{C W S, r e f} \in[285,295] K$.

- $\dot{m}_{C H W S} \in\{0\} \bigcup[20,235] \mathrm{kg} / \mathrm{s}$.

- $T_{C H W S, r e f} \in[276.5,280.4] K$.

- $T_{C H W R} \in[283,295] K$.

- $Z_{b} \in[0.1,1] z_{\text {tank }}$.

\section{G. Model Summary}

By collecting Equations (3)-(6), and also descretizing the system with sampling time of 1 hour, the dynamic equations can be compacted as following:

$$
\begin{aligned}
& x(t+1)=f(x(t), u(t), d(t)) \\
& y(t)=g(x(t), u(t), d(t)) ;
\end{aligned}
$$

where

$$
\begin{aligned}
& f= \begin{cases}f_{1}(x(t), u(t), d(t)) ; & \text { if } \dot{m}_{C H W S} \leq \dot{m}_{c m p} \\
f_{2}(x(t), u(t), d(t)) ; & \text { if } \dot{m}_{C H W S}>\dot{m}_{c m p}\end{cases} \\
& u(t)=\left[T_{C W S, r e f} ; \dot{m}_{C H W S} ; T_{C H W S, r e f}\right] \in \mathbb{U} \\
& x(t)=\left[U_{a} ; U_{b} ; z_{a} ; z_{b}\right] \\
& d(t)=\left[\dot{m}_{c m p, s}\right] \in \mathbb{D}(t) \\
& y(t)=\left[T_{C H W R} ; z_{b}\right] \in \mathbb{Y} .
\end{aligned}
$$




\begin{tabular}{lll}
\hline SUMMER & Period A & (May 1st though Oct. 31st) \\
\hline Peak & $12: 00-18: 00$ & except holidays \\
Partial-peak & 8:30-12:00 & except holidays \\
& AND 18:00-9:30 & \\
Off-peak & 21:30-8:30 & Mon. through Fri. \\
& ALL DAY & Sat., Sun, and holidays \\
\hline \hline WINTER & Period B & (Nov. 1st though Apr. 30st) \\
\hline Partial-peak & $8: 30-21: 30$ & except holidays \\
Off-peak & 21:30-8:30 & Mon. through Fri. \\
& ALL DAY & Sat., Sun, and holidays \\
\hline
\end{tabular}

TABLE IV: Definition of time periods

\begin{tabular}{ll}
\hline Total Demand Rates (\$ per kW) & \\
\hline Maximum Peak Demand Summer & $\$ 12.40$ \\
Maximum Part-Peek Demand Summer & $\$ 2.74$ \\
Maximum Demand Summer & $\$ 7.52$ \\
Maximum Part-Peak Demand Winter & $\$ 1.04$ \\
Maximum Demand Winter & $\$ 7.52$ \\
\hline
\end{tabular}

TABLE V: Total Demand Rates

\begin{tabular}{ll}
\hline Total Energy Rates $(\$$ per kWh) & \\
\hline Peak Summer & $\$ 0.13593$ \\
Part-Peak Summer & $\$ 0.09204$ \\
Off-Peak Summer & $\$ 0.07392$ \\
Part-Peak Winter & $\$ 0.08155$ \\
Off-Peak Winter & $\$ 0.07118$ \\
\hline
\end{tabular}

TABLE VI: Total Energy Rates

$\mathbb{U}$ is the feasible control input set defined in Section II-F; $\mathbb{Y}$ is the feasible output set defined in Section II-F; $\mathbb{D}(t)$ refers to the time-variant disturbances ranges and is defined in Figure 3.

\section{H. Energy Price}

The energy price mainly refers to the unit price of the electricity power consumed by the chilling system. UC Merced is currently enrolled in a special plan, electric schedule E-20, which is designated for customers with maximum demands of 1000 kilowatts or more. The customer's monthly charge for the service under Schedule E-20 is the sum of a customer charge, demand charges, and energy charges, and all the unit price varies depending on the period of time. Table IV shows the definition of the time periods. The customer charge is a flat monthly fee, which is a constant independent of time. Schedule E-20 has three demand charges, a maximum-peak-period demand charge, a maximum-partpeak-period demand charge, and a maximum-demand charge. The maximum-peak-period-demand charge per kilowatt applies to the maximum demand during the month's peak hour, the maximum-part-peak-demand charge per kilowatt applies to the maximum demand during the months part-peak hours, and the maximum-demand charge per kilowatt applies to the maximum demand at any time during the month. Table $\mathrm{V}$ lists all the demand rates according to the specific period.

The energy charge is the sum of the energy charges from the peak, partial-peak, and off-peak periods. We denote the unit electricity price defined by Table $\mathrm{V}$ as the function $C(t)$. The customer pays for energy by the kilowatt hour $(\mathrm{kWh})$. The detailed energy rates refers to Table VI

\section{MPC PROBLEM FORMULATION}

This section presents the design of a MPC control whose objective is to find the optimal control sequence so that we can satisfy the required cooling load while receiving the lowest electricity bills. Consider the following optimization problem:

$$
\begin{aligned}
J^{\star}(x(t), t)= & \min _{\hat{u}_{0 \mid t}, \cdots, \hat{u}_{M-1 \mid t}} \sum_{i=1}^{N-1}\left\{\left\|C(t+i) E\left(x_{i \mid t}, u_{i \mid t}\right)\right\|_{S}\right. \\
& \left.+\left\|u_{i \mid t}\right\|_{R}\right\} \\
\text { s.t. } & y_{i \mid t} \in \mathbb{Y}, \forall i=1,2, \cdots, N \\
& u_{i \mid t} \in \mathbb{U}, \forall i=1,2, \cdots, N \\
& y_{N \mid t} \in \mathbb{Y}_{f}(t) ; \\
& E\left(x_{i \mid t}, u_{i \mid t}\right)=\operatorname{Power}\left(x_{i \mid t}, u_{i \mid t}\right) \Delta T \\
& {\left[u_{0 \mid t}^{\prime}, \cdots, u_{N-1 \mid t}^{\prime}\right]^{\prime}=B \otimes I_{m}\left[\hat{u}_{0 \mid t}^{\prime}, \cdots, \hat{u}_{M-1 \mid t}^{\prime}\right] } \\
& x_{k+1 \mid t}=f\left(x_{k \mid t}, u_{k \mid t}, d(k)\right) ; \\
& y_{k \mid t}=g\left(x_{k \mid t}, u_{k \mid t}, d(k)\right) ; \\
& d(k) \in \mathbb{D}(k), \forall k=1,2, \cdots, N
\end{aligned}
$$

where $\mathbb{Y}_{f}(t)$ is the terminal constraint set, $C(t)$ is the energy price at time $t, \Delta T=1$ hour, $S$ is the weight on the energy consumption, and $R$ is the weight of the control inputs.

Let $U_{t \rightarrow t+N \mid t}^{\star}=\left\{u_{t \mid t}^{\star}, \cdots, u_{t+N-1 \mid t}^{\star}\right\}$ be the optimal solution of Problem (9) at time $t$, and $J_{t}^{\star}(x(t))$ the corresponding value function. Then, the first element of $U_{t \rightarrow t+N \mid t}^{\star}$ is implemented to the system (7):

$$
u(t)=u_{t \mid t}^{\star}
$$

The optimization Problem (9) is repeated at time $t+1$, based on the new state $x_{t+1 \mid t+1}=x(t+1)$, yielding a moving or receding horizon control strategy. The proposed MPC controller uses a move blocking strategy to reduce the computational time required for its real time implementation, which will be detailed later. The control sampling time is one hour, and prediction horizon is set to $24 \mathrm{~h}$ (one day).

\section{A. Dual Stage Optimization}

Problem (9) is a MINLP (Mix Integer Nonlinear Program), whose complexity limits real time implementation. We solve the Problem (9) by using a tailored branch and bound strategy. In the first stage, we choose the tank operation mode profile, and in the second stage, with a fixed tank operation mode, the problem is recast to a NLP (nonlinear program) problem which can be solved by optimization packages available such as NPSOL [12].

In this paper, the results presented use a fixed tank operation mode profile shown in Figure 4, where 1 means charging mode and -1 indicates discharging mode. 


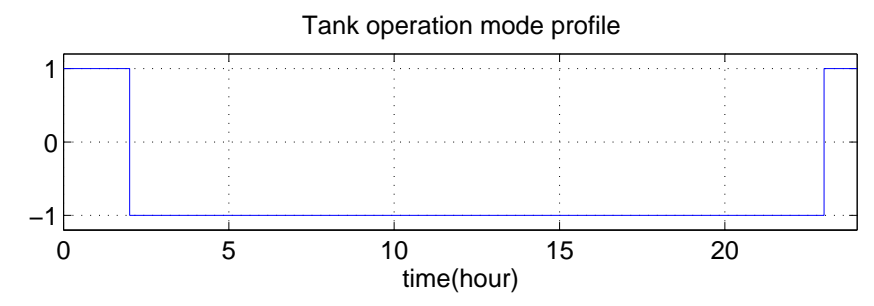

Fig. 4: Tank Operation Mode Profile

\section{B. Move Blocking Strategy}

The prediction horizon of the proposed MPC controller is 24 hours, and the control sampling time is one hour. As a result, there would be total 72 optimization variables as the control input dimension is 3 . It is common practice to reduce the degrees of freedom by fixing the input or its derivatives to be constant over several time steps [19]. In this paper, we are using the Moving Window Blocking approach proposed in [20], We first need the following definitions before providing the algorithm used.

Definition 1 (Admissible Blocking Matrix): A matrix $B \in\{0,1\}^{N \times M}$ is an admissible blocking matrix if $M<N$, and one entry in each row of $\mathrm{B}$ is equal to 1 , the elements of the matrix are arranged in an "upper staircase" form, i.e. if the column in which a 1 occurs in the i'th row is

$$
j^{\star}(i):=\left\{j \mid B_{i, j}=1\right\}
$$

then $j^{\star}(i+1) \geq j^{\star}(i)$ for all $i \in\{1,2, \cdots, N-1\}$. Where $B_{i, j}$ denotes the element of $\mathrm{i}$ 'th row and $\mathrm{j}$ 'th column of matrix B.

Definition 2 (Blocking Length Vector): Given an admissible blocking matrix $B \in\{0,1\}^{N \times M}$, the blocking length vector $\mathrm{L}(\mathrm{B})$ is defined as the columnwise summation of the matrix B. An admissible block vector corresponds to a unique blocking length matrix.

The following algorithm is in the proposed MPC.

Algorithm 1 (Moving Window Blocking): Given an initial blocking length matrix $L_{0}$;

1) let $\mathrm{i}=0$;

2) if $L_{i}(1)>1$,

$$
\begin{aligned}
& L_{i+1}:=L_{i} \\
& L_{i+1}(1):=L_{i}(1)-1 \\
& L_{i+1}(\text { end }):=L_{i}(\text { end })+1 .
\end{aligned}
$$

$$
\text { if } L_{i}(1)=1 \text {, }
$$

$$
L_{i+1}:=\left[L_{i}(2: e n d), L_{i}(1), 0\right] .
$$

3) if $L_{i}=L_{0}$, stop. Otherwise, go to next step;

4) let $i:=i+1$, and go to step 2 .

Where $L_{i}(e n d)$ is the last element of $L_{i}$.

By following Algorithm 1, we can get a series of blocking length matrices for each step. In this work, we choose $L_{0}=$ $[2,2,18,1,1,0]$, and Algorithm 1 will give

$$
\begin{aligned}
L_{1} & =[1,2,18,1,1,1] \\
L_{2} & =[2,18,1,1,2,0] \\
L_{3} & =[1,18,1,1,2,1] \\
L_{4} & =[18,1,1,2,2,0] \\
\vdots & \\
L_{24} & =[2,2,18,1,1,0]
\end{aligned}
$$

\section{Terminal Constraints}

It is well known that stability and feasibility are not ensured by the MPC law without terminal cost or terminal constraints. Usually the problem is augmented with a terminal cost and a terminal constraint set $\mathbb{Y}_{f}$. Typically $\mathbb{Y}_{f}$ is a robust control invariant set which guarantees that if Problem (9) is feasible for a given $x_{0}$, then it is always feasible for $t \geq 0$.

A formal definition of robust control invariant sets follows.

Definition 3 (Robust Control Invariant Set): A set $\mathcal{C} \subseteq \mathbb{X}$ is said to be a robust control invariant set for system (7) if for every

$x(k) \in \mathcal{C}, \exists u(k) \in \mathbb{U} \mid f(x(k), u(k), d(k)) \subseteq \mathcal{C} \quad \forall d(k) \in \mathbb{D}$

The set $\mathcal{C}_{\infty}$ is said to be the maximal robust control invariant if it is robust control invariant and contains all robust control invariant set contained in $\mathbb{X}$. Where $f(x(k), u(k), d(k))$ is defined in Equation (7).

A treatment of sufficient conditions which guarantees persistent feasibility of MPC problems goes beyond the scope of this work and can be found in the surveys [18]; Since the disturbance is periodic, the idea proposed by F. Blanchini and W. Ukovich in [4] can be applied to the proposed MPC controller. The invariant sets, if it exists, will be time variant and periodic with the same period as the disturbances.

In order to guarantee that the tank has enough cold water to satisfy the demand, we use the algorithm proposed in [4] to calculate the CPI (Controlled Periodic Invariant) sets for the system described in Equations (5a). The system is a simple buffer plant subject to constraints in Section II-F and periodic disturbance modeled in Figure 3.

We implemented the algorithm proposed in [4] and Figure 5 plots the lower bound of the computed periodic set $\mathbb{Y}_{f}(t)$. If the height of the cooler water in the tank is greater than the lower bounds, there exists a feedback control law that will satisfy any disturbance belonging to the envelope in Figure 3 without violating the states and inputs constraint.

\section{SIMULATION RESULTS}

Define the one day Electricity Bill as

$$
\text { Bill }=\sum_{t=0}^{t=24} \operatorname{Pr}(t) \operatorname{Power}\left(x_{t}, u_{t}\right) \Delta T
$$

where $\Delta T$ is the sampling time (1 hour).

Next we simulate system (7) in closed loop with the MPC controller (9), (7) and compare the results with current 


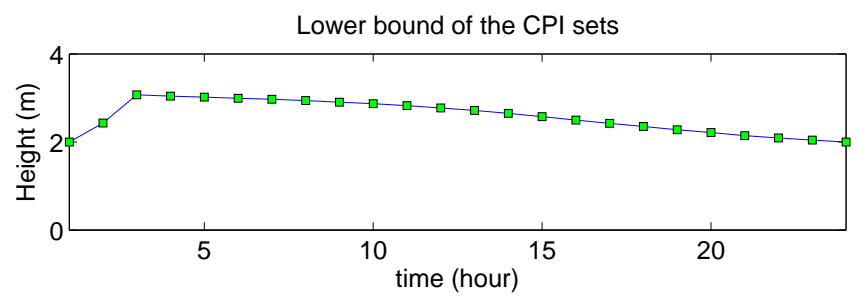

Fig. 5: CPI sets of the system

manual operation over 7 days. The load of the campus is chosen to be the maximum of the load shown in Figure 3 in order to test the robustness of the controller. The temperature of wet bulb is extracted from the historical data and will be replaced by the data from weather stations in future work.

1) Current Manual Operation: The cooling system in UC Merced is operated manually with following control input sequences. The temperatures set-points $T_{C W S \text {, ref }}$ and $T_{C H W S, r e f}$ are kept constant to $286.26 \mathrm{~K}$ and $277.04 \mathrm{~K}$ respectively, and the chiller will be $\mathrm{ON}$ around $10 \mathrm{pm}$ to $2 \mathrm{am}$ every two days to charge the tank to full with a constant mass flow rate of $138 \mathrm{Kg} / \mathrm{s}$ and the chiller is off the rest of time. Simulation results plotted in Figure 6 shows that the system converges to a periodic operation. The tank will is charged to full during the night, and discharged at daytime. Based on Equation (12), the average bill in one day for this heuristic control sequence is $\$ 174.54$.

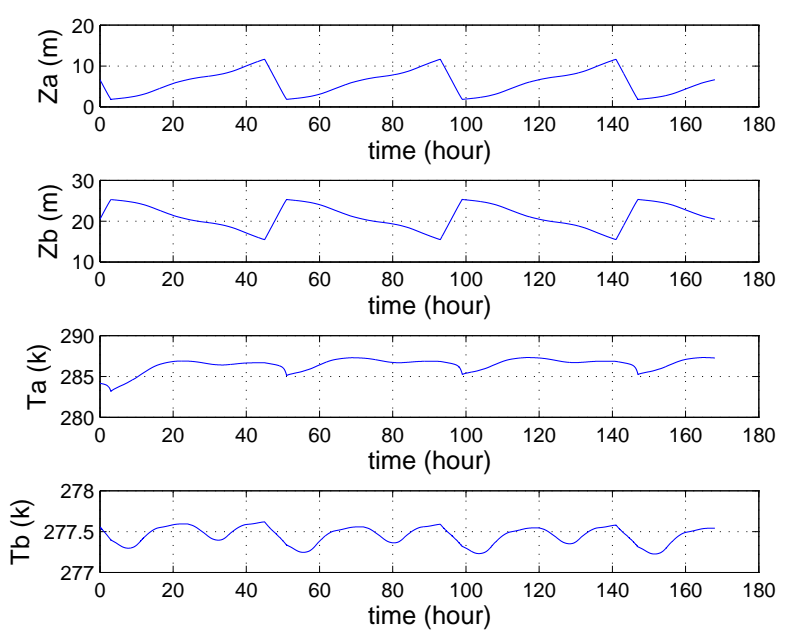

Fig. 6: Simulation results of the heuristic controller

2) Operation With MPC Controller: Simulation results plotted in Figure 7 shows that the system converges to a periodic operation. Figure 8 reports the control inputs of heuristic control logic and MPC controller. The system states evolution is shown in Figure 7. We can observe that the height and the temperature of the cold water in the tank behaves periodically over the time. The optimal MPC policy does not charge the tank to the full capacity an thus avoiding tank losses. Instead, the tank is preferably charged to $35 \%$ of the tank volume to achieve better efficiency. Also, the
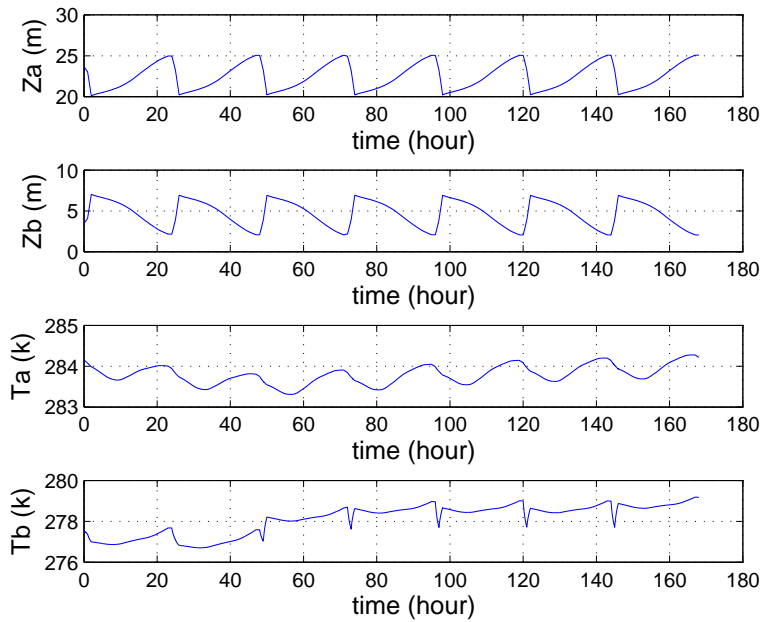

Fig. 7: Simulation result of MPC controller

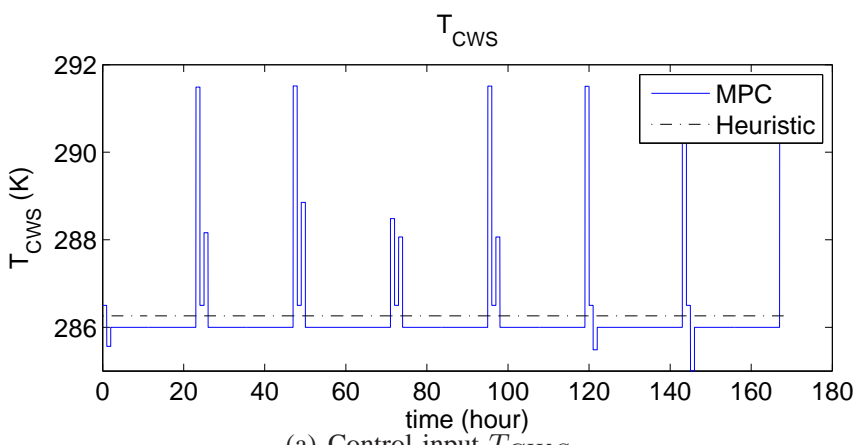

(a) Control input $T_{C W S}$

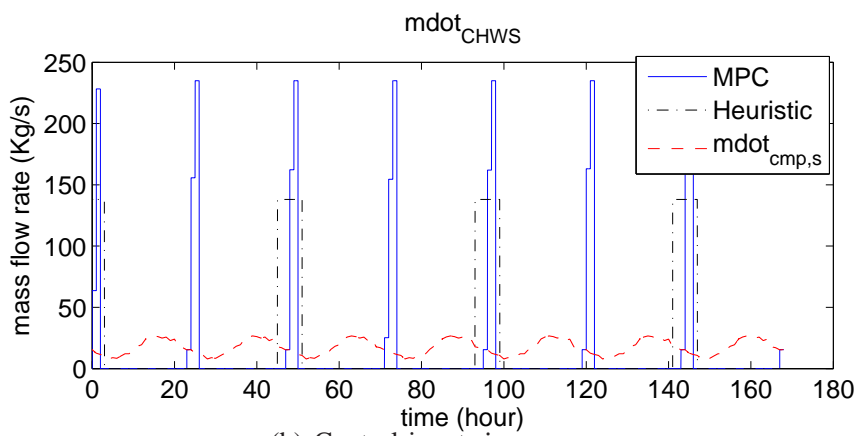

(b) Control input $\dot{m}_{C H W S}$

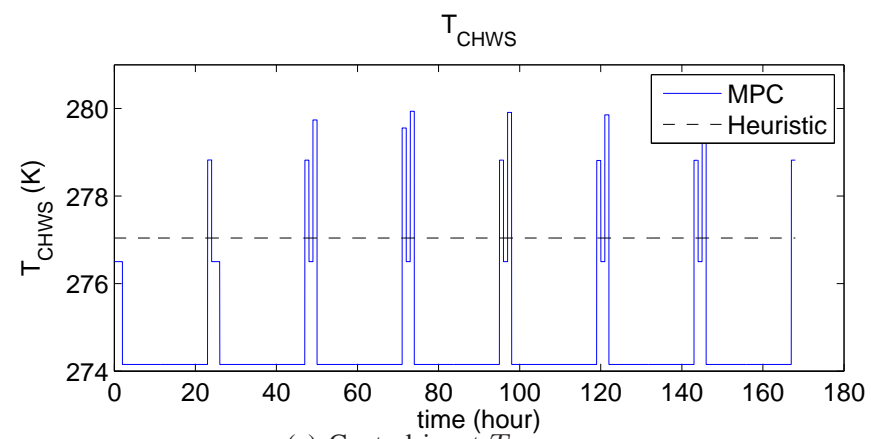

(c) Control input $T_{C H W S}$

Fig. 8: MPC Control Sequence 
resulting bill under the MPC policy (at periodic steady state) in one day is $\$ 131.71$, which corresponds to a saving of $24.5 \%$ compared to the heuristic controller.

\section{CONCLUSIONS}

In this paper, we introduced a simplified model of the chilling system at UC Merced and a Model Predictive Control algorithm. Periodic invariant sets, moving block strategy and dual stage optimization have been used to tackle complexity and feasibility issues of the resulting scheme. Preliminary simulation results showed that the daily electricity bill can be reduced of $24.5 \%$ compared to the current heuristic manual control sequence. The results is very promising and current work is focusing on model validation in order to confirm that a similar range of performance improvement could be obtained for the actual plant. In particular the building load model requires further investigation and validation. Our near future work will also involve experimental validation of the proposed scheme at UC Merced.

\section{ACKNOWLEDGMENTS}

This work was partial supported by the Department of Energy and Laurence Berkeley National Laboratories. We thank Philip Haves, John Elliott, Satish Narayanan, Stella M. Oggianu, Brian Coffey and Michael Wetter for constructive and fruitful discussions on the system modeling and control implications.

\section{REFERENCES}

[1] 2008. https://gaia.lbl.gov/virBui.

[2] 2008. https://gaia.lbl.gov/bcvtb.

[3] S. Domenech A. Ponsich, C. Azzaro-Pantel and L. Pibouleau. Mixedinteger nonlinear programming optimization strategies for batch plant design problems. Industrial \& Engineering Chemistry Research, 46(3):854-863, 2007.

[4] F. Blanchini and W. Ukovich. Linear programming approach to the control of discrete-time periodic systems with uncertain inputs. $J$. Optim. Theory Appl., 78(3):523-539, 1993.

[5] F. Borrelli. Constrained Optimal Control of Linear and Hybrid Systems, volume 290. Springer-Verlag, 2003.

[6] F.C. Winkelmann W.F. Buhl Y.J. Huang C.O. Pedersen R.K. Strand R.J. Liesen D.E. Fisher M.J. Witte D.B. Crawley, L.K. Lawrie and J. Glazer. Energyplus: creating a new-generation building energy simulation program. Energy and Buildings, 33(4):319 - 331, 2001.

[7] C.V. Rao D.Q. Mayne, J.B. Rawlings and P.O.M. Scokaert. Constrained model predictive control: Stability and optimality. 36(6):789814, June 2000.

[8] S.J. Qin J.B. Rawlings F. Allgöwer, T.A. Badgwell and S.J. Wright. Nonlinear predictive control and moving horizon estimation - and introductory overview. 1999.

[9] C. Felsmann G.P. Henze and G. Knabe. Evaluation of optimal control for active and passive building thermal storage. International Journal of Thermal Sciences, 43(2):173 - 183, 2004.

[10] M. Krarti G.P. Henze and M.J. Brandemuehl. Guidelines for improved performance of ice storage systems. Energy and Buildings, 35(2):111 $-127,2003$.

[11] S. Herkel G.P. Henze, J. Pfafferott and C. Felsmann. Impact of adaptive comfort criteria and heat waves on optimal building thermal mass control. Energy and Buildings, 39(2):221 - 235, 2007.

[12] K. Holmstrom. The tomlab optimization environment in matlab. Adv. Model. Optim, 1:47, 1999.

[13] M. Morari J.H. Lee and C.E. Garcia. State-space interpretation of model predictive control. 1992.

[14] M. Koschenz and V. Dorer. Interaction of an air system with concrete core conditioning. Energy and Buildings, 30(2):139 - 145, 1999.
[15] M. Koschenz and B. Lehmann. Development of a thermally activated ceiling panel with pcm for application in lightweight and retrofitted buildings. Energy and Buildings, 36(6):567 - 578, 2004.

[16] J. Lee. Mixed-integer nonlinear programming: some modeling and solution issues. IBM J. Res. Dev., 51(3):489-497, 2007.

[17] S. Liu and G.P. Henze. Experimental analysis of simulated reinforcement learning control for active and passive building thermal storage inventory: Part 1. theoretical foundation. Energy and Buildings, 38(2): 142 - 147, 2006.

[18] D.Q. Mayne. Constrained optimal control. European Control Conference, Plenary Lecture, September 2001.

[19] S.J. Qin and T.A. Badgwell. An overview of industrial model predictive control technology. pages 232-256, 1997.

[20] E.C. Kerrigan R. Cagienard, P. Grieder and M. Morari. Move blocking strategies in receding horizon control. Journal of Process Control, $17(6): 563-570,2007$. 